\title{
Experimental Study of Software Testing Strategies used in Mobile Testing
}

\author{
Monika Soni \\ President, \\ Auricle Global Society of Education and Research, India. \\ 12.monika@gmail.com
}

Abstract: - This paper will explain the meaning of mobile testing as well as mobile application testing. This will also discuss about the testing strategies which will be used for mobile testing. There are advantages as well as disadvantages of the mobile testing. There are many job opportunities in the field of mobile testing. Mobile testing can also be manual as well as automated. There are many tools available for mobile testing. This paper will give brief idea about the types of automated tools used for mobile testing.

Keywords: - Mobile Testing, Advantages, Disadvantages, Mobile Testing techniques.

Introduction: - Mobile application testing is the process of testing all the applications which are designed for the mobile devices. They are tested for their functionality, compatibility and also usability. The mobile testing can be manual as well as automated. It is very important that all the applications that are developed for use on mobile must be tested on mobile devices before they are made available for downloading. It is important because once they are made available for downloading then there are chances of getting errors. So, it should be tested whether they are working and giving expected results. They should also be tested for their compatibility across various platforms. Once all this is done then only, they should be made available for the customers to download it.

Mobile Testing Life Cycle: - The process which involves various number of phases or steps which must be completed in order to test the mobile is called as Mobile Testing Life Cycle. There are certain steps and all the steps are important to follow in order to identify the bugs of mobile application and to make sure that the application works efficiently. This can be understood with the help pf the following diagram: [1]

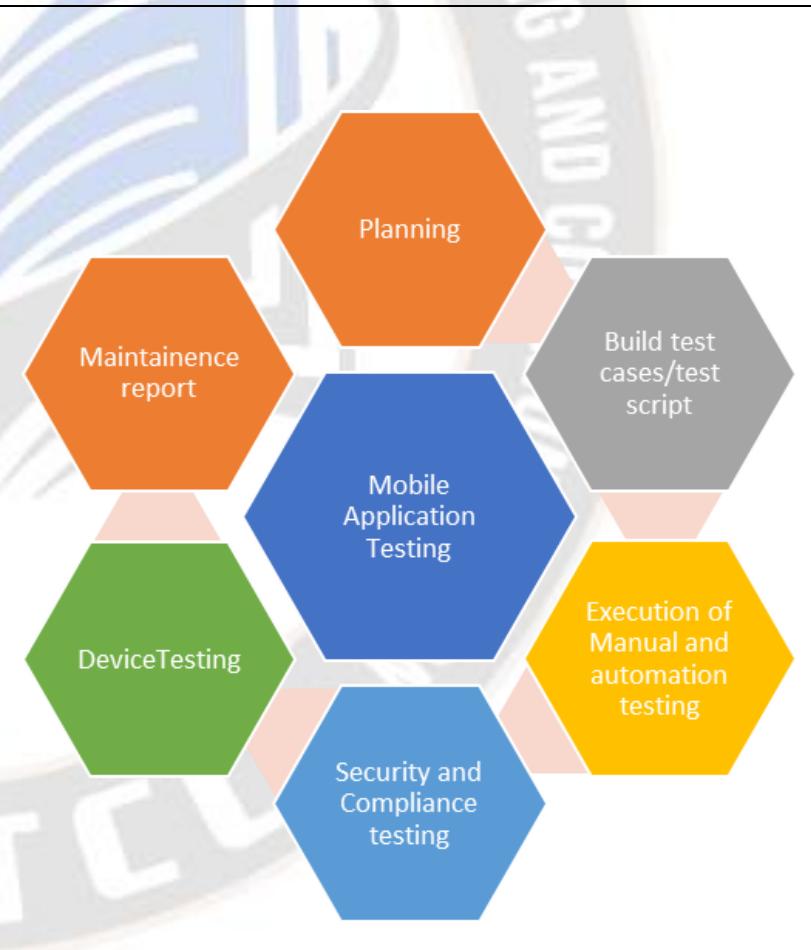

Figure 1 Different phases of Mobile Application Testing

It is very important the testers are involved during the development phase of any mobile application being developed. As in this scenario, the testers may have better understanding of the applications being made and can also give their feedback regarding their intuition of upcoming errors. This way most of the future bugs can be eliminated during the development phase itself. 
a. Planning: - During this phase, based on the development document, the testing team prepares their own test document and plan about how to proceed with their testing process. They will consider all the scenarios about which the test cases must be build and executed. They should consider the following points: -

- Whether the application under development is compatible with the devices or not?

- Will the application interact with other applications without giving any errors?

- How the application will handle the stress and load under extreme conditions and whether it will give good performance under all such conditions?

- Is the application is working as per the expected functions.

All the above points will be discussed and updated in the documents. It is expected from all the testers to keep updating the document during the complete duration of application testing process.

b. Build Test Cases/Test Scripts: - As the name suggests, the testers involved starts writing the test cases as well as the test scripts based on the document of the previous phase. In this stage it is also decided that which components of the application will be tested manually and which components to be tested automation. Based on these separate documents are developed which includes test cases for manual testing and also the test scripts for automation testing. It is also be identified which are the reused automated test scripts. Everything should be mentioned in the respective documents without fail.

c. Execution of Manual and Automation testing scripts: In this phase the actual execution of the test cases and test scripts is done. As it is already identified in the previous stage as to which are the test cases and the test scripts. All are executed and test for any errors. These can be done parallel or one after the other.

d. Security and Compliance Testing: - It is the most important phase as this will make sure that the applications developed for the mobiles are completely safe to use and there will not be any disclosure of private and confidential data of the users. The users will also check the application for the safety to make sure that their data will remain safe and there are no threats to them, only then they will be interested to install the applications. In this phase it is also tested whether all the policies issued by government is followed for the applications being made.

e. Device Testing: - It is also important to test the devices on which the applications will be used. So, all the test cases as well as the test scripts should be executed on all the devices in order to identify the bugs.

f. Maintenance Report: - In this phase whatever is tested and followed throughout the testing process will be documented in summary report. If there are any new changes that are made then that also must be included in the summary report.

Hence, all the phases are important to be followed and identified for any bugs. If there are any bugs then they must be rectified before the application is available for downloading so that the users do not face any issues.

Challenges of Mobile Testing: -[2]

There many challenges faced by the Mobile Application testers. These are explained a s following: -

1. Different Mobile Devices: - These days there are wide variety of mobile devices available as a result of which it is challenging task for the testers to test the applications on all the devices. Sometimes due to lack of availability of the devices at the same time, it is difficult to test the applications.

2. Compatibility: - It is also very difficult to test the compatibility of the application on many devices due to the lack of the availability of the devices. One application might be compatible on same browser but not on same devices.

3. Lack of availability: - The testing process gets delayed under some cases like the lack of availability of the devices to the testers. This will increase the testing time and hence the efficiency cannot be achieved.

4. Downloading issues:- Sometimes on some devices it is very difficult to download the application. Some times the application may take longer than usual which in turn decrease the efficiency with which the tester works.

5. Connection issues: - There are different speed rates provided by the various network providers and it is also seen that few mobile devices can support only one particular type of connection. Under such cases also it gets difficult for the mobile testers to test the applications.

6. Power conception issues: - There are certain types of applications which consumes large amount of power of the device battery. So, it is also important that the testers test all these attributes before the application is made available for the end users.

7. Device Settings: - There are many inbuilt properties of the devices which allows the settings of the device to be changed. Thus, it is challenging task for the mobile testers to see those settings and make sure that the settings do not change whether the application is installed or not 
8. Testing methods: - There are two types of testing the mobile applications:

- Real devices

- Emulators

Now the challenge is that the testing of mobile application on the emulators will not give efficient testing results as it might miss few points while testing the application. Hence, good testing results can only be achieved on real devices which are sometimes very costly to buy.

Testing methodology in Mobile application testing: -[3]

Following are the types of testing which are done in Mobile application testing:

1. Functional Testing

2. Performance and Load Testing

3. Compatibility Testing

4. Usability Testing

5. Security Testing

6. Manual Testing

7. Automation Testing

8. Localisation Testing

9. Installation Testing

10. Device Testing

Each one of the above is explained in detail as following: -

1. Functional Testing: - This type pf testing is done to determine whether the application is working as expected and giving the results which, it is supposed to give. This testing tests that all the functions which are supposed to be done by that particular application is being carries out without any errors or not. Following are the important points which should be kept in mind while doing functional testing of the applications: -

- To test whether the user can login in to the application without any difficulties

- To make sure that the login credentials are kept safely.

- The application responds if a certain action is done. For example, if the user is trying to login with correct credentials then it should allow him to login without any errors.

- For all this the tester first of all should gather information regarding what they are supposed to test and how they will test it.

- The testers are then supposed to make test cases or test scripts once they know what to test and how to test (manual testing/automation testing).

- Once it is determined which cases to execute manually and which to test using automation then the testers should run the test cases in real environment of the device.

- The testers then should rectify all the bugs which may have come after the execution of the test cases in the previous step.

- The testers should also maintain the records of all the steps and test cases and whatever is done during the testing of the mobile application testing for future references.

2. Performance and Load Testing: - This testing is also one of the most important type of testing of mobile application testing. Without conducting this testing, it is not advisable to deploy the applications for downloading for the use of the clients. In this type of testing, it is determined how the application works under extreme load conditions as well as normal conditions. Also, it is tested how the application performs across various network available and on different mobile devices and on different platforms. Following are the challenges while doing performance and load testing of the mobile application testing: - [4]

- The challenges that tester might have that he is not sure whether he will be able to do performance testing efficiently due to lack of devices available to him

- As, he will have emulators installed to test on almost all types of devices, it will not give proper results as it is kind of simulator. The test result of emulators might be different from those of testing in real device.

- The process is time consuming as there is challenge of location of the device used by the user which will extend the testing time longer than usual.

3.Compatibility testing: - The tester tests the compatibility of the application on different devices. The compatibility testing will test whether the particular application is working as expected on all types of platforms, browsers, etc. The usability testing is related to the look and feel of the application but compatibility testing means testing it across all platforms etc. It is important that the compatibility testing is also done during the development of the application to make sure that it will not give future errors. This testing is necessary to conduct as if it is not done then there are chances that the application might give many errors once it is live and it will result this into failure of the application.

4. Usability Testing: - This testing means the look and feel of the application being developed. This checks whether the application is easy to use and user friendly. Following are the steps involved in usability testing: - [5]

* Test Objective and plan: - In this step the testers decide what they are supposed to test and then sets the objective of the 
testing process. This will include what they want to test and how they will conduct the tests.

*Identify the tasks: -Once the objectives of the test are decided then small test tasks are made based on the document of the previous phase. The test users should then follow all the small tasks assigned to them and check for any errors.

*Test document: - In order to conduct test of the application consent form is being filled by the user testers and also questionnaire form. There are many documents which are prepared while doing usability testing.

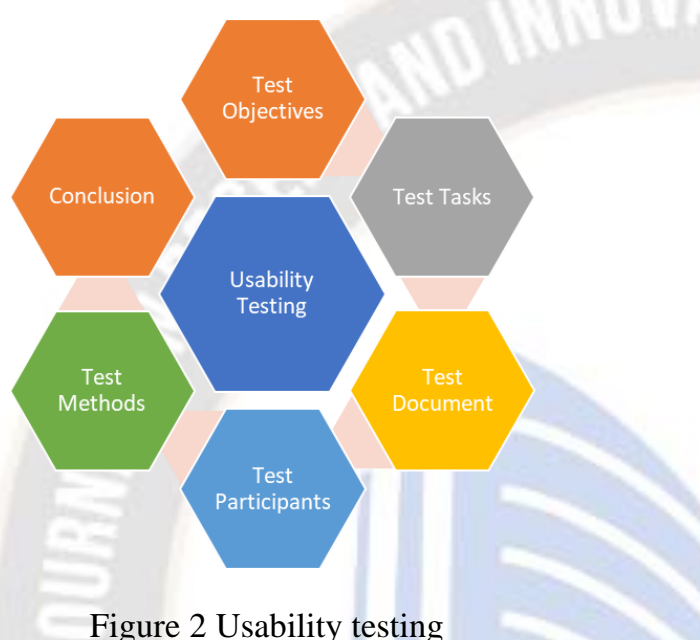

Figure 2 Usability testing

*Test Participants: - The people involved in testing the application are known as test participants and has following roles and responsibilities to do: -

* First of all, a consent form is signed with the test participants.

*The test participants must be available for the usability testing all the time.

*They should have the device on which the application is supposed to be tested.

*Test Methods: - There are basically two types of methods of testing usability testing: -

a. Remote Testing: -This is done remotely without involving the end users.

b. Lab based testing: - In this type of testing the testing is done involving the real customers on real devices.

5. Security Testing: - This type of testing is very important to conduct to avoid threat to security of the data of the users. Although, the security of mobile applications is more, safe as compared to the web- based applications, still it is very important to conduct this step. Following are the points that should be kept in mind while doing the security testing: -
* Security of the data stored in an application.

*Authorisation and authentication security.

*Ensuring the security of communication.

*Security on various platforms on which the application is executed.

6. Manual Testing: - The applications that are developed for mobile devices can be tested manually. For this it is important for the testers to be involved during the development of the application so that they have the complete knowledge of the application being developed and helps them to predict errors. Based upon the information gathered they can decide which components of the mobile application can be tested manually. Based on that they can create test cases and execute them manually in order to find the bugs.

Advantage of manually testing the Mobile applications: -

- These are cost effective as it is done manually.

- It is easy way of testing the components of the mobile applications.

\section{Disadvantages: -}

- The test cases cannot be reused.

- The process of testing becomes slow as it takes a lot of time to test the applications manually.

7. Automation Testing: - The components of mobile application can be tested automation also which saves a lot of time. Based on the information gathering the testers can decide which components of the applications can be tested via automation. So, then they can create the test scripts. But for this they must be having complete knowledge of the automation tools and should make sure that the automation tools are easily available to them for executing the test scripts.

Advantages of Automation Mobile application testing: -

- It saves a lot of time of testing process.

- Few test scripts can be reused after doing modifications.

- $\quad$ Cost effective

Disadvantages of automation mobile application testing: -

- The major drawback is that if the tester does not have the knowledge of the automation testing tool then this process cannot be used.

- The tools for automation are costly so not everyone can use it.

8.Localisation testing: -This type of testing is done to make sure that the application works without any errors in different 
regions and locations. For example, if the language of the application is changed still it should function properly. The two main things that are considered in localisation testing is to test the user interface and the content. SO if anybody selects any particular language then the content of the user interface should also be in that selected language and also all the functions of the application must work with full accuracy as it is supposed to do.

9. Device Testing: - The mobile applications must be tested on various devices which has mobility and also on different platforms and environments. There are emulators which has dummy of real devices but still it will not give the same results which it will give on real time devices.

10.Installation testing: - The applications should also be tested on the basis of its installation process. It should be tested that the application must be installed without any difficulty on all the devices. The installation process should be easy and with all the instructions given by the team to the end users so that he can himself install the application without any difficulty.

Tools used for Mobile Application Testing: -

There are many numbers of tools used for mobile application testing. Few of them can be seen in the following diagram: -

\section{Test
Complete}
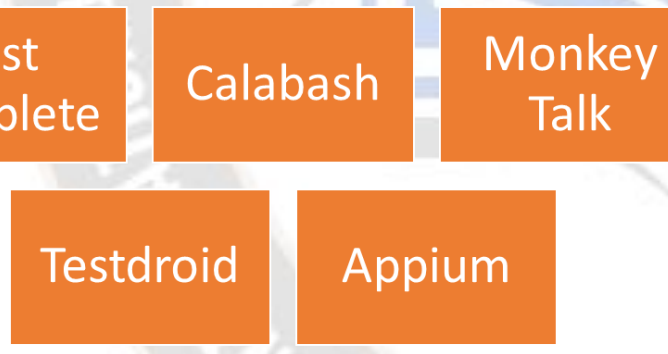

Figure 3 Testing tools for Mobile Application testing

Advantages of testing tools for Mobile Application testing: -

- Using these tools makes the process easy. It saves a lot of time and energy.

- It is easy to communicate the test results using the testing tools.

- The time spent in doing manual documentation can be saved.

- If there is new member in the team then he can refer to the testing tool in order to understand the process used for this application testing.

Disadvantages of testing tools used for mobile application testing: -
- $\quad$ The person should have sound knowledge of the tools being used only then they will be able to get the true results.

- The cost of maintaining these tools is high and so not all the companies can afford them.

- In some cases, the process takes a lot of time to test the applications.

- Testing cannot be done if the sources are not available throughout the testing process.

Conclusion: - Hence, it is concluded that there are challenges as well as using mobile applications. The testers of the mobile application must be involved during the development process of the applications as that will make their task easy for testing the application. It is also seen that there are many types pf testing that must be conducted for the mobile applications before the application is made available for the users to install.

\section{References: -}

1.https://dzone.com/articles/your-step-by-step-mobileapplication-testing-proce

2.https://dzone.com/articles/your-step-by-step-mobileapplication-testing-proce

3.https://testlio.com/blog/10-mobile-testing-types-andapproaches/

4.https://performancelabus.com/mobile-appperformance-testing/

5.https://usabilitygeek.com/usability-testing-mobileapplications/ 